Research Article

\title{
Prescription audit study in a tertiary care hospital using the anatomical therapeutic chemical and defined daily dose classification concept
}

\author{
Ajay Kumar Gupta ${ }^{1 *}$, Siddhartha Mishra ${ }^{2}$
}

${ }^{1}$ Department of Pharmacology, Armed Forces Medical College, Pune, Maharashtra, India, ${ }^{2}$ Department of Medicine, Command Hospital, Southern Command, Pune, Maharashtra, India

Received: 14 August 2014 Revised: 03 September 2014 Accepted: 06 September 2014

*Correspondence to: Dr. Ajay Kumar Gupta, Email: ajayneera2007@ rediffmail.com

Copyright: (C) the author(s), publisher and licensee Medip Academy. This is an openaccess article distributed under the terms of the Creative Commons Attribution NonCommercial License, which permits unrestricted noncommercial use, distribution, and reproduction in any medium, provided the original work is properly cited.

\begin{abstract}
Background: In 1996, WHO recognized the need to develop and use the anatomical therapeutic chemical (ATC) and defined daily dose (DDD) as an international standard for utilization studies wherein it appointed experts in clinical pharmacology, drug utilization, drug regulation, drug evaluation, statistics, and medicine to monitor drug utilization on a standard and uniform pattern around the world.

Methods: All the prescriptions presented to main dispensary of the hospital from January-December 2013 in mid-month period of 10 days were taken for the analysis in terms of pharmacovigilance, polypharmacy, drug usage group wise, pharmacoeconomics, and drug utilization in DDD/1000/day along with ATC classification using customized software.

Results: 35,808 prescription forms containing 121,303 drugs were presented to main dispensary of the hospital across which medicines were dispensed. Polypharmacy was calculated to be $3.27 \pm 0.44$ standard deviation. Approximately, $60 \%$ of total prescription forms analyzed were lacking essential and basic such information as age, gender, or diagnosis. Pharmacoeconomics of this study indicates that a major budget was mainly spent on drug groups belonging to - metabolic, antibiotics, hormones, and related drugs. A voluminous consumption of drugs belonged to drug groups - metabolic drugs like statins, hormones, and related drugs such as various oral hypoglycemics, anti-hypertensives, psychotropics, and proton pump inhibitors or $\mathrm{H}_{2}$ blockers, all indicative toward stressful and sedentary lifestyle of modern era and unhealthy lifestyles, a trend of increasing morbidity and mortality, reducing significantly the productive man-hours.

Conclusions: This study was undertaken in a government tertiary care hospital which gives insight into the day to day functioning status of our health care delivery system. It is an opportunity on self-assessment in further improving the health care delivery by implementing measures which economizes on scarce health care budget as well as minimizing the common prescription errors.
\end{abstract}

Keywords: Defined daily dose use, Drug utilization, Pharmacovigilance, Prescription audit

\section{INTRODUCTION}

Quality assessment of medical care rendered to patients is the need of time and can only be assessed by a prescription audit, because the same is based on documentary evidence which supports elements of prescription writing having there in diagnosis, treatment, and justified details. In principle, it is prudent for physicians to have an objective and systemic way of analysis of treatment and care provided to the patient. ${ }^{1}$ Thus, medical audit may be defined as looking at what one is doing with the aim of providing patient care and use of resources. ${ }^{2}$ Prescription audit is a tool designed for the objective assessment by and to the medical care provider of meeting their own standards in their professional day to day care. Hence, prescription audit is a tool as well as a technique and its application is science as well as an art.

In order to measure drug use, it is important to have both a classification system and a unit of measurement which preferably should be standardized and acceptable to all the agencies in the world for uniform comparisons of results. This need was formulated in the late sixties, which still was in the infancy period in the field of drug utilization research. Due to an increasing interest in this field, a symposium entitled The Consumption of Drugs took place in Oslo in 1969 organized by the WHO Regional Office for Europe. 
It was at this symposium the Drug Utilization Research Group was established tasked with the development of internationally applicable methods for drug utilization research. ${ }^{4}$

Inspired by this interest, a system named anatomical therapeutic chemical (ATC) classification was developed by the Norwegian Medicinal Depot. Norwegian researchers also developed a technical unit of measurement called the defined daily dose (DDD) to be used in drug utilization studies. $^{5}$ The ATC/DDD system has been used in Norway since the early seventies for presenting drug consumption data.

In 1996, when the decision on globalization the ATC/DDD system was taken, the WHO Division of drug Management and Policies in Geneva established the WHO International Working Group for Statistics Methodology. This working group now consists of $12 \mathrm{WHO}$ appointed experts in clinical pharmacology, drug utilization, drug regulation, drug evaluation, statistics, and medicine. The members of the working group represent different users of the ATC/DDD system and different nationalities as they represent the 6 WHO global regions. The following countries are represented in the working group: Australia, Japan, India, Pakistan, Morocco, Ghana, Zimbabwe, USA, Argentina, The Netherlands, and Sweden. The working group meets twice annually. The WHO Collaborating Centre for Drug Statistics Methodology receives expert advice from the working group and acts as secretariat for the group.

In developing countries, the constantly increasing number of medicine list and treatment option serves to increase the irrational medicine use and cost that ultimately leads to poor patient outcome and significant wastage of money and resources. Cost of each such irrational drug use is enormous in terms of both scarce resources and adverse clinical consequences of therapies that may have real risks but no objective benefits. Now a days, the prescribing pattern is changing and has become just an indication for the medicine with some instructions of doses without considering its rationality. ${ }^{6}$ The study of prescribing pattern is that part of medical audit which seeks to monitor, evaluate, and if necessary suggest modifications in prescribing practices of medical professionals so as to make medical care rational and cost effective. ${ }^{7,8}$ Prescription audit also forms part of drug utilization studies which audits prescribing indicators such as average consultation time, average time for dispensing, if all drugs actually are dispensed, drugs if adequately labeled/ legibly written, patients' knowledge if has understood the usage of drugs as told by pharmacist and facility indicators like availability of copy of essential drugs list/drug formulary of hospital, VED analysis etc. ${ }^{9}$

Prescription is an order to the pharmacist for scientific medication to the patient at a particular time for the disorder summarized for logical diagnosis. ${ }^{10}$ This focuses at the diagnostic acumen and therapeutic proficiency of the clinician with the necessary instruction for palliation or restoration of the patient's health. ${ }^{12}$ Thus, quality-of-life can be improved by enhancing the standards of medical treatment at all levels of health care delivery systems. ${ }^{13}$ Medical audit overseas the observance of these standards. Setting standards and assessing the quality of medical care through performance review should become part of everyday clinical practice. ${ }^{14}$

In India, several research groups have initiated efforts to study the drug utilization patterns mostly in the last three decades. Most of these groups are from pharmacology departments of medical institutions, pharmacy colleges, newly established pharmacy practice departments, and other independent agencies.

Various studies in different parts of the World conducted on the prescription auditing ${ }^{15-18}$ produced their own database for the future comparative study. Study of drug prescription and its utilization in India have been reported at both hospital ${ }^{19-21}$ and community levels..$^{22,23}$ Most of these studies have measured drug utilization by counting of prescriptions which indicates actually prescribing frequency of drug prescription. To have uniformity of drug utilization studies at various places, DDD is of great value as compared to traditional units of measurements of drugs utilization by weight or cost. DDD is of great value as compared to traditional units of measurement of drugs utilization by weight or cost. Indian studies have used DDD concept and focused on drug utilization in selected groups of patients for a brief period. ${ }^{24-26}$ We undertook this study at one of the tertiary care hospitals for a period of 1 year based on the prescriptions presented to dispensary and classified the drugs by ATC/DDD methodology to determine the prevalence of prescribing pattern of all major groups of drugs. An attempt was also made to associate prescription data with two demographic characteristics, viz, age and sex, to develop patient population database.

\section{Statistics}

Student's t-test was applied as a measure of dispersion and other figures are in percentage being a descriptive study.

\section{METHODS}

Study place: Tertiary Care Government Hospital, Pune.

Study design: Prospective descriptive study.

Ethical consideration: Approved from Institutional Ethical Committee.

Study duration: 01 January, 2013-31 December, 2013. 


\section{Methodology}

Prescription forms which were presented to the dispensary of the hospital by the patients for collecting their medicines from January 2013 to December 2013 in the mid-month period of 10 days, i.e., from $11^{\text {th }}$ to $20^{\text {th }}$ of each month were taken into account for analysis. Since the period of study was one full calendar year, the data were consolidated every 03 monthly, i.e., Jan-Mar, Apr-Jun, Jul-Sep, Oct-Dec.

\section{RESULTS}

\section{Pharmacovigilance}

All prescriptions from $11^{\text {th }}$ to $20^{\text {th }}$ of each month were counted and analyzed for their completeness. The prescriptions which were incomplete on account of lacking elementary parameters such as age, gender, and diagnosis were labeled incomplete. Further, the prescription forms were also categorized into having generic or brand name of drugs in them. This was also further categorized as prescriptions originating from specialists, residents, and general duty medical practitioners (Tables 1-4).

\section{Polypharmacy}

All ages of patients were categorized into eight categories as 0-10, 11-20, 21-30, 31-40, 41-50, 51-60, 61-70, and $>70$ year. The prescriptions forms having different number of drugs in them were tabulated to analyze the most common age group of people having the highest number of drugs prescribed per prescription (Figures 1-4).

\section{Drug usage group wise}

All ages of patients were categorized into eight categories as above. The drugs in the prescription forms were subdivided into nine major categories, viz. cardiovascular (CVS), nonsteroidal anti-inflammatory drugs (NSAIDs) including analgesics and antipyretics, anti-microbial agents (AMA), central nervous system, gastrointestinal (GIT), respiratory system and anti-allergics, hormones and related drugs, nutrition and vitamins, and lastly metabolic group of drugs. This gave an indication of the highest prescribed group of drugs in particular age group of patients (Figures 5-8).

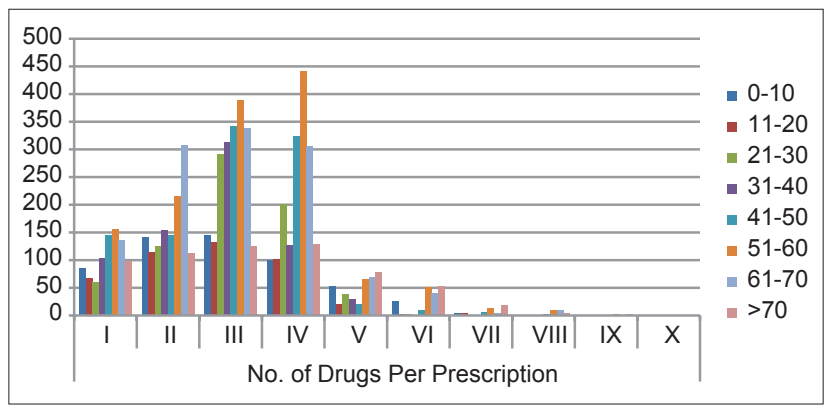

Figure 1: Polypharmacy for period: January 2013-March 2013.

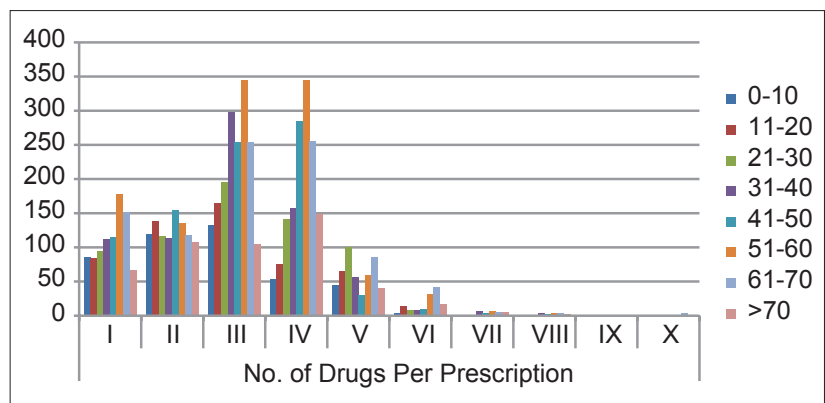

Figure 2: Polypharmacy for period: April 2013-June, 2013.

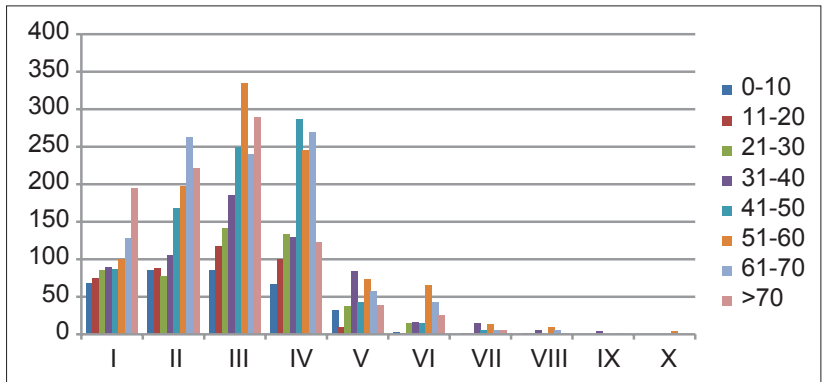

Figure 3: Polypharmacy for period: July 2013-September, 2013

Table 1: Pharmacovigilance for period: January 2013-March 2013.

\begin{tabular}{|c|c|c|c|}
\hline Total prescription slips & 9654 & Contains & 32464 drugs \\
\hline \multirow{2}{*}{ Prescription slips complete } & 3625 & Not complete & 6029 \\
\hline & $\begin{array}{c}\text { Specialist }(\%) \\
n=3803(63.08)\end{array}$ & $\begin{array}{l}\text { Residents }(\%) \\
n=2149(35.64)\end{array}$ & $\begin{array}{l}\text { GDMP (\%) } \\
n=77(1.28)\end{array}$ \\
\hline Prescriptions lacking age & $1399(36.79)$ & $883(41.09)$ & $39(50.47)$ \\
\hline Prescriptions lacking gender & $383(10.07)$ & $241(11.21)$ & $13(16.88)$ \\
\hline Prescriptions lacking diagnosis & $3572(93.93)$ & $2028(94.37)$ & $70(90.91)$ \\
\hline Prescriptions in brand name & $4160(36.21)$ & $2007(34.87)$ & $58(34.12)$ \\
\hline Prescription in generic name & $7330(63.79)$ & $3749(65.13)$ & $110(64.71)$ \\
\hline
\end{tabular}

GDMP: General duty medical practitioners 
Table 2: Pharmacovigilance for period: April 2013-June 2013.

\begin{tabular}{|c|c|c|c|}
\hline Total prescription slips & 8832 & Contains & 29392 drugs \\
\hline Prescription slips complete & 3104 & Not complete & 5728 \\
\hline & $\begin{array}{c}\text { Specialist }(\%) \\
n=3574(61.92)\end{array}$ & $\begin{array}{l}\text { Residents }(\%) \\
n=2162(37.74)\end{array}$ & $\begin{array}{l}\text { GDMP (\%) } \\
n=19(0.33)\end{array}$ \\
\hline Prescriptions lacking age & $775(21.85)$ & $367(16.98)$ & $4(21.05)$ \\
\hline Prescriptions lacking gender & $304(8.57)$ & $141(6.52)$ & $3(15.79)$ \\
\hline Prescriptions lacking diagnosis & $3219(90.75)$ & $2063(95.42)$ & $18(94.74)$ \\
\hline Prescriptions in brand name & $3885(35.81)$ & $1925(33.13)$ & $12(33.33)$ \\
\hline Prescription in generic name & $6963(64.19)$ & $3886(66.87)$ & $24(66.67)$ \\
\hline
\end{tabular}

GDMP: General duty medical practitioners

Table 3: Pharmacovigilance for period: July 2013-September 2013.

\begin{tabular}{|c|c|c|c|}
\hline Total prescription slips & 8557 & Contains & 28916 drugs \\
\hline \multirow[t]{2}{*}{ Prescription slips complete } & 3389 & Not complete & 5168 \\
\hline & $\begin{array}{l}\text { Specialist (\%) } \\
n=3469(67.12)\end{array}$ & $\begin{array}{l}\text { Residents }(\%) \\
n=1659(32.10)\end{array}$ & $\begin{array}{l}\text { GDMP (\%) } \\
n=40(0.77)\end{array}$ \\
\hline Prescriptions lacking age & $883(24.02)$ & $377(22.70)$ & $13(32.10)$ \\
\hline Prescriptions lacking gender & $128(3.69)$ & $78(4.70)$ & $5(12.50)$ \\
\hline Prescriptions lacking diagnosis & $3260(93.98)$ & $1592(95.96)$ & $37(92.50)$ \\
\hline Prescriptions in brand name & $4166(34.35)$ & $1599(31.61)$ & $33(37.08)$ \\
\hline Prescription in generic name & $7961(65.65)$ & $3460(68.39)$ & $55(61.80)$ \\
\hline
\end{tabular}

GDMP: General duty medical practitioners

Table 4: Pharmacovigilance for period: October 2013-December 2013.

\begin{tabular}{|c|c|c|c|}
\hline Total prescription slips & 8765 & Contains & 30531 drugs \\
\hline \multirow{2}{*}{ Prescription slips complete } & 3280 & Not complete & 5485 \\
\hline & $\begin{array}{c}\text { Specialist (\%) } \\
n=3467(63.21)\end{array}$ & $\begin{array}{l}\text { Residents }(\%) \\
n=2003(36.52)\end{array}$ & $\begin{array}{l}\text { GDMP (\%) } \\
n=15(0.27)\end{array}$ \\
\hline Prescriptions lacking age & $1011(29.16)$ & $521(26.01)$ & $7(46.48)$ \\
\hline Prescriptions lacking gender & $174(5.02)$ & $100(4.99)$ & $2(13.33)$ \\
\hline Prescriptions lacking diagnosis & $3271(94.35)$ & $1915(95.61)$ & $14(93.33)$ \\
\hline Prescriptions in brand name & $3986(34.67)$ & $1834(31.87)$ & $13(39.39)$ \\
\hline Prescription in generic name & $7510(65.33)$ & $3920(68.13)$ & $20(60.61)$ \\
\hline
\end{tabular}

GDMP: General duty medical practitioners

\section{Gender wise usage of drug groups}

Furthermore, a comparison of drug groups' usage was done for eight age groups as above to know the morbidity pattern in the population (Tables 5-8).

\section{Pharmacoeconomics}

Drugs' price was taken from the procurement cost of our hospital which is, of course, much lower than the retail price as printed on the drug packages. An estimate was made of the cost factor involved on health care based on drug groups, as above, in the four quadrants of a year for 10 days of each month (Figures 9 and 10).

\section{Drug utilization}

All the drugs which are considered in the study are classified using ATC concept and their consumption pattern in all the four quadrants are expressed in conventional pattern of percentage as well as scientifically in accordance of WHO recommendations using DDD concept. The recommended formula by WHO for drug utilization pattern is (Table 9):

Total number of dosage strengths of units prescribed $\times$ each dosage $\mathrm{DDD} / 1000 /$ day $=\frac{\text { unit } \times 1000}{\mathrm{DDD} \times \text { Duration of study } \times \text { Total }}$ sample size 


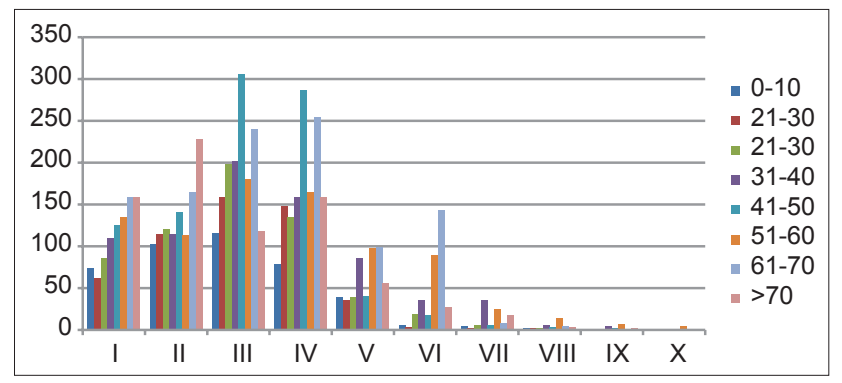

Figure 4: Polypharmacy for period: October 2013-December 2013.

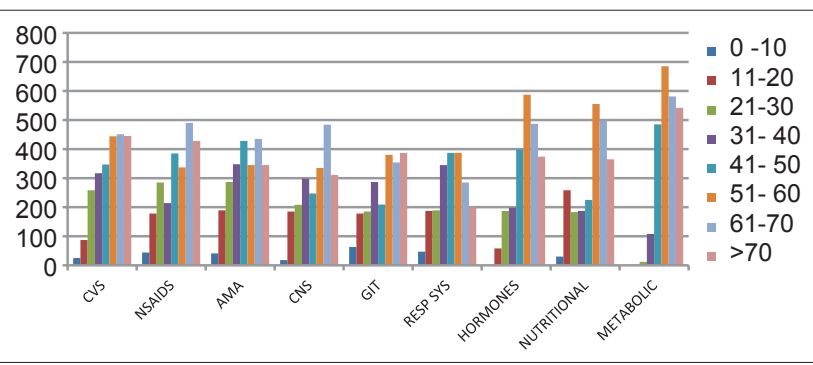

Figure 5: Drug usage groupwise for the period: January 2013-March 2013.

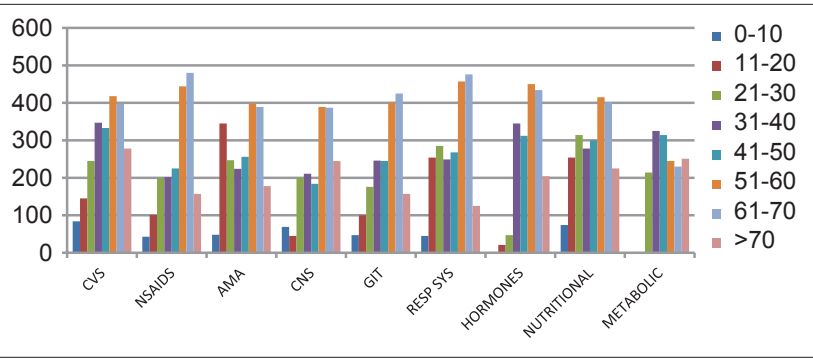

Figure 6: Drug usage groupwise for the period: April 2013-June 2013.

Table 5: Genderwise prescribing frequency for drug groups for a period: January 2013-March 2013.

\begin{tabular}{|c|c|c|}
\hline \multirow[t]{3}{*}{ Drug groups } & \multicolumn{2}{|c|}{$\begin{array}{l}\text { Number of patients } \\
\text { (\% of the population) }\end{array}$} \\
\hline & Male & Female \\
\hline & $n=5480(60.77)$ & $\mathrm{n}=3537(\mathbf{3 9} .23)$ \\
\hline CVS & $2100(38.32)$ & $1000(28.87)$ \\
\hline NSAIDs & $1987(36.26)$ & 1117 (31.58) \\
\hline AMA & $1748(31.90)$ & $1018(28.78)$ \\
\hline CNS & $1348(24.60)$ & $797(22.53)$ \\
\hline GIT & $1254(22.88)$ & $666(18.83)$ \\
\hline $\begin{array}{l}\text { Respiratory system } \\
\text { and anti-allergics }\end{array}$ & $1645(30.02)$ & $983(27.79)$ \\
\hline $\begin{array}{l}\text { Hormones and } \\
\text { related drugs }\end{array}$ & $1452(26.50)$ & $837(23.66)$ \\
\hline Nutritional & $2458(44.85)$ & $1469(41.53)$ \\
\hline Metabolic & $1648(30.07)$ & $765(21.63)$ \\
\hline
\end{tabular}

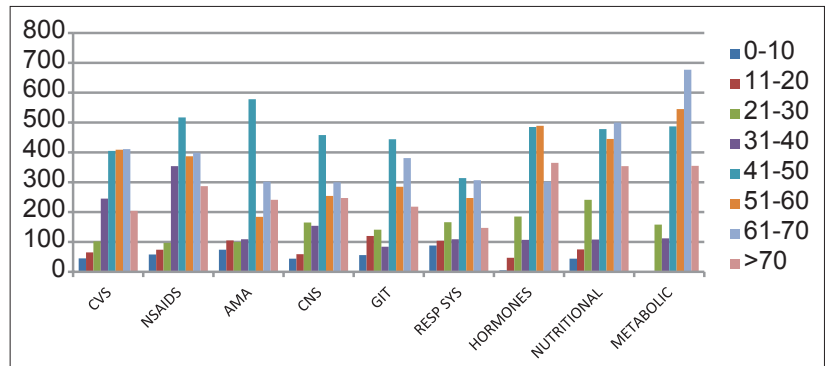

Figure 7: Drug usage groupwise for the period: July 2013-September 2013.

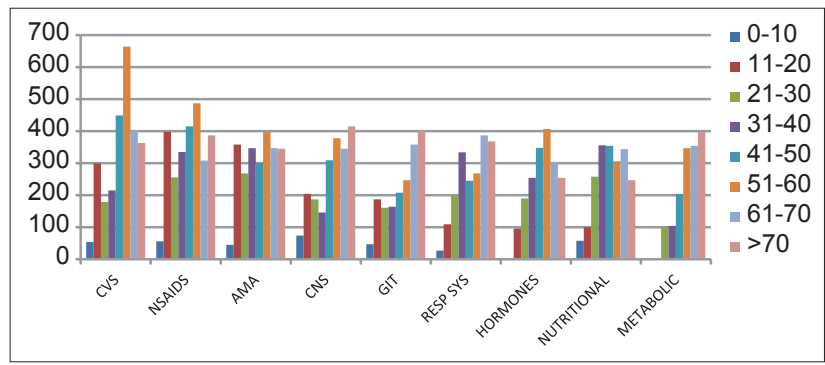

Figure 8: Drug usage group wise for the period: October 2013-December 2013.

Table 6: Genderwise prescribing frequency for drug groups for a period: April 2013-June 2013.

\begin{tabular}{|lcc|}
\hline Drug groups & \multicolumn{2}{c|}{$\begin{array}{c}\text { Number of patients } \\
\text { \% of the population) }\end{array}$} \\
\cline { 2 - 3 } & $\begin{array}{c}\text { Male } \\
\mathbf{n = 5 4 8 0}(\mathbf{6 0 . 7 7 )})\end{array}$ & $\begin{array}{c}\text { Female } \\
\mathbf{n}=\mathbf{3 5 3 7}(\mathbf{3 9 . 2 3})\end{array}$ \\
\hline CVS & $1645(36.74)$ & $1048(27.75)$ \\
\hline NSAIDs & $1437(32.10)$ & $1164(29.79)$ \\
\hline AMA & $1478(33.01)$ & $1106(28.31)$ \\
\hline CNS & $1141(25.49)$ & $854(21.86)$ \\
\hline GIT & $1314(29.35)$ & $1101(28.18)$ \\
\hline $\begin{array}{l}\text { Respiratory system } \\
\text { and anti-allergics }\end{array}$ & $1393(31.12)$ & $1070(27.39)$ \\
\hline $\begin{array}{l}\text { Hormones and } \\
\text { related drugs }\end{array}$ & $1154(25.78)$ & $759(19.43)$ \\
\hline Nutritional & $1648(36.81)$ & $1435(36.73)$ \\
\hline Metabolic & $1125(25.13)$ & $836(21.40)$ \\
\hline
\end{tabular}

CVS: Cardiovascular, NSAIDs: Nonsteroidal anti-inflammatory drugs, AMA: Anti-microbial agents, CNS: Central nervous system, GIT: Gastrointestinal

\section{DISCUSSION}

A prescription is a prescribers order to dispense a specific treatment - Usually, medication for a specific patient. Thus, it needs no further emphasis that for error-free dispensing, all prescription order forms should be legible, unambiguous, dated and signed with a rubber stamp of prescriber bearing his/her registration number for an optimal communication between prescriber, pharmacist, and nurse/health care providers. Many common errors include lack of patients 
Table 7: Genderwise prescribing frequency for drug groups for a period: July 2013-September 2013.

\begin{tabular}{|lcc|}
\hline Drug groups & \multicolumn{2}{c|}{$\begin{array}{c}\text { Number of patients } \\
\text { (\% of the population) }\end{array}$} \\
\cline { 2 - 3 } & $\begin{array}{c}\text { Male } \\
\mathbf{n = 4 5 7 8}(\mathbf{5 9 . 5 1 )}\end{array}$ & $\begin{array}{c}\text { Female } \\
\mathbf{n}=\mathbf{3 1 1 5}(\mathbf{4 0 . 4 9 )}\end{array}$ \\
\hline CVS & $2054(44.87)$ & $1112(35.70)$ \\
\hline NSAIDs & $1987(43.40)$ & $1190(38.20)$ \\
\hline AMA & $1648(36.00)$ & $1184(38.01)$ \\
\hline CNS & $1456(31.80)$ & $865(27.77)$ \\
\hline GIT & $1289(28.16)$ & $873(28.03)$ \\
\hline $\begin{array}{l}\text { Respiratory system } \\
\text { and anti-allergics }\end{array}$ & $1485(32.44)$ & $1034(33.19)$ \\
\hline $\begin{array}{l}\text { Hormones and } \\
\text { related drugs }\end{array}$ & $1647(35.98)$ & $949(30.47)$ \\
\hline Nutritional & $1875(40.96)$ & $1263(40.55)$ \\
\hline Metabolic & $1568(34.25)$ & $910(29.21)$ \\
\hline
\end{tabular}

CVS: Cardiovascular, NSAIDs: Non-steroidal anti-inflammatory drugs, AMA: Anti-microbial agents, GIT: Gastrointestinal, CNS: Central nervous system

Table 8: Genderwise prescribing frequency for drug groups for period: October 2013-December 2013.

\begin{tabular}{|lcc|}
\hline Drug groups & \multicolumn{2}{c|}{$\begin{array}{c}\text { Number of patients } \\
\text { \% of the population) }\end{array}$} \\
\cline { 2 - 3 } & $\begin{array}{c}\text { Male } \\
\mathbf{n}=\mathbf{4 0 1 7}(\mathbf{5 5 . 5 9 )})\end{array}$ & $\begin{array}{c}\text { Female } \\
\mathbf{n}=\mathbf{3 2 0 9}(\mathbf{4 4 . 4 1 )})\end{array}$ \\
\hline CVS & $2154(47.05)$ & $871(27.96)$ \\
\hline NSAIDs & $2147(46.90)$ & $1268(40.71)$ \\
\hline AMA & $1987(43.40)$ & $912(29.28)$ \\
\hline CNS & $1789(39.08)$ & $906(29.09)$ \\
\hline GIT & $1487(32.48)$ & $662(21.25)$ \\
\hline $\begin{array}{l}\text { Respiratory system } \\
\text { and anti-allergics }\end{array}$ & $1547(33.79)$ & $1003(32.20)$ \\
\hline $\begin{array}{l}\text { Hormones and } \\
\text { related drugs }\end{array}$ & $1385(30.25)$ & $694(22.28)$ \\
\hline Nutritional & $1854(40.50)$ & $1132(36.34)$ \\
\hline Metabolic & $1466(32.02)$ & $748(24.01)$ \\
\hline
\end{tabular}

CVS: Cardiovascular, NSAIDs: Non-steroidal anti-inflammatory drugs, AMA: Anti-microbial agents, GIT: Gastrointestinal, CNS: Central nervous system

particular such as self or relative, age, gender in the superscription whereas diagnosis, dosage and frequency of drug administration are other few. Prescribing in a generic name offers the pharmacist flexibility in selecting drug or substitutes of the same in case of possibility of near similar drug if available. However, it should not be assumed that every generic drug product is as satisfactory as the trade-named product, though most generics are satisfactory. Bioavailability for the products varies between manufacturers and sometimes between different lots of drug produced by the same manufacturer. It thus produces

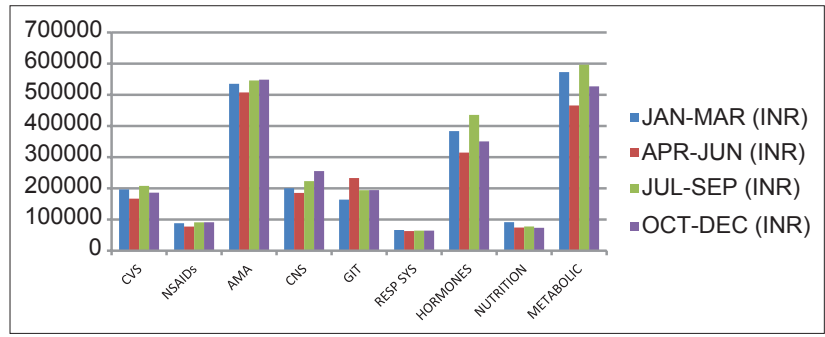

Figure 9: Pharmacoeconomics - drug group wise: January 2013-December 2013.

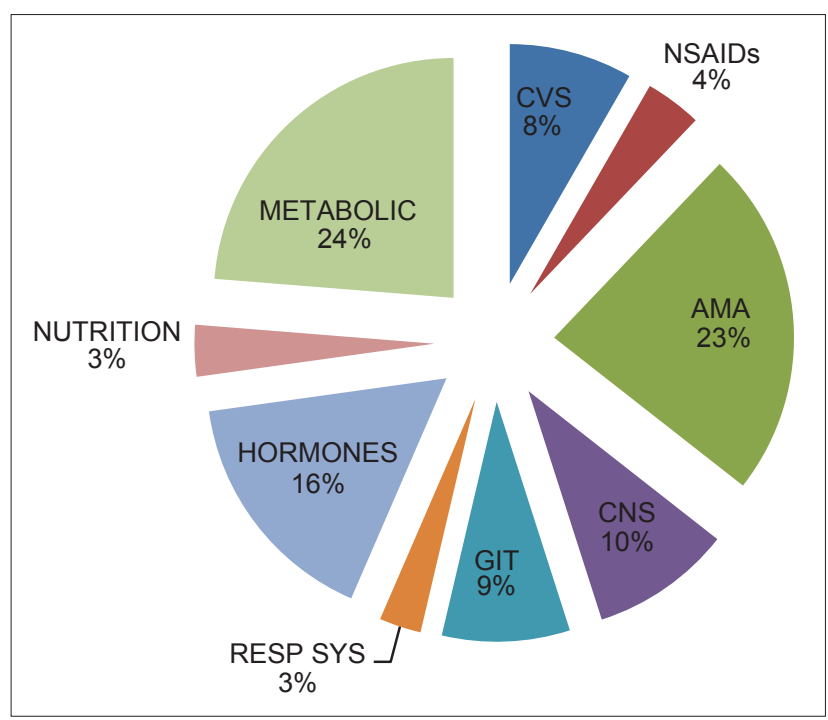

Figure 10: Pharmacoeconomics - expense on various drug groups (\%): January 2013-December 2013.

same drug but having different biochemical equivalence which is specifically relevant for drugs having a narrow therapeutic margins and some chronic drug therapies like various psychiatric agents and anti-epileptics, etc. Thus, in case of very small number of drugs which usually have a low therapeutic index, poor solubility or a high ratio of inert ingredients to active drug content, a specific manufacturer's product may give a more consistent result. In this study, it was found that approximately two-third of prescriptions was in generic name and rest in brand names optimizing the balance between the two.

Average number of drugs per prescription form is an important index of prescription audit as it forms the basis for review and educational intervention in day to day prescribing practices of doctors. It is preferable to keep the mean number of drugs per prescription as low as possible since higher figures may lead to increased risk of drug interactions, as well as unnecessary economic burden. Most importantly for antimicrobials, such overuses increase the chance of bacterial resistance. In this study, we have found average three drugs per prescription form which is within acceptable limits. Other studies based on community practices reveal a mean of two drugs per prescription form. ${ }^{27}$ Some hospital-based studies in India reported a figure of 3-5 drugs per prescription form. ${ }^{19-21}$ By and large, it is difficult to keep the number of drug per prescription 
Table 9: Drug utilization in percentage and DDD/1000/day.

\begin{tabular}{|c|c|c|c|c|c|c|c|c|c|}
\hline \multirow[t]{2}{*}{ Drug } & \multirow[t]{2}{*}{ ATC } & \multicolumn{2}{|c|}{ Jan-Mar } & \multicolumn{2}{|c|}{ Apr-Jun } & \multicolumn{2}{|c|}{ Jul-Sep } & \multicolumn{2}{|c|}{ Oct-Dec } \\
\hline & & $\%$ & DDD & $\%$ & DDD & $\%$ & DDD & $\%$ & DDD \\
\hline \multicolumn{10}{|l|}{ Category: CVS } \\
\hline Acenocoumarol & B01AA07 & 0.15 & 0.46 & 0.17 & 0.51 & 0.18 & 0.57 & 0.15 & 0.45 \\
\hline Acetazolamide & S01EC01 & 0.06 & 0.32 & 0.05 & 0.23 & 0.06 & 0.30 & 0.06 & 0.32 \\
\hline Amiodarone & C01BD01 & 0.04 & 0.29 & 0.04 & 0.28 & 0.04 & 0.32 & 0.05 & 0.39 \\
\hline Amlodipine & C08CA01 & 1.50 & 49.77 & 1.49 & 48.80 & 1.15 & 38.31 & 1.53 & 50.37 \\
\hline Aspirin & $\mathrm{B} 01 \mathrm{AC} 06$ & 0.69 & 11.39 & 0.67 & 10.92 & 0.68 & 11.25 & 0.65 & 10.66 \\
\hline Atenolol & C07AB03 & 1.33 & 14.73 & 1.32 & 14.36 & 1.30 & 14.36 & 1.51 & 16.51 \\
\hline Carvedilol & C07AG02 & 0.27 & 1.11 & 0.30 & 1.22 & 0.28 & 1.16 & 0.27 & 1.11 \\
\hline Clonidine & $\mathrm{C} 02 \mathrm{AC} 01$ & 0.04 & 0.14 & 0.04 & 0.14 & 0.05 & 0.16 & 0.05 & 0.16 \\
\hline Clopidogrel & B01AC04 & 0.27 & 8.86 & 0.25 & 8.13 & 0.27 & 9.14 & 0.27 & 8.86 \\
\hline Colspirin & B01AC06 & 0.26 & 8.59 & 0.27 & 8.73 & 0.27 & 8.86 & 0.27 & 8.86 \\
\hline Digoxin & C01AA05 & 0.14 & 2.23 & 0.10 & 1.48 & 0.12 & 1.85 & 0.14 & 2.13 \\
\hline Diltiazem & C08DB01 & 0.14 & 0.03 & 0.13 & 0.02 & 0.16 & 0.03 & 0.13 & 0.03 \\
\hline Dipyridamole & $\mathrm{B} 01 \mathrm{AC} 07$ & 0.01 & 0.05 & 0.02 & 0.11 & 0.02 & 0.10 & 0.02 & 0.13 \\
\hline Enalapril & C09AA02 & 1.05 & 17.32 & 1.00 & 16.34 & 1.05 & 17.38 & 1.03 & 16.95 \\
\hline Ethamsylate & B02BX01 & 0.15 & 7.36 & 0.11 & 5.65 & 0.18 & 9.00 & 0.13 & 6.64 \\
\hline Felodipine & C08CA02 & 0.27 & 5.91 & 0.21 & 4.52 & 0.30 & 6.54 & 0.26 & 5.72 \\
\hline Frusemide & C03CA01 & 1.21 & 40.09 & 1.17 & 38.25 & 1.29 & 42.95 & 1.20 & 39.44 \\
\hline Hydrochlorthiazide & C03AA03 & 0.06 & 0.95 & 0.07 & 1.13 & 0.09 & 1.50 & 0.05 & 0.90 \\
\hline Indapamide & C03BA11 & 0.14 & 2.16 & 0.12 & 1.83 & 0.19 & 2.99 & 0.13 & 2.00 \\
\hline Isopropamide & A03AB09 & 0.02 & 0.41 & 0.03 & 0.45 & 0.04 & 0.61 & 0.03 & 0.42 \\
\hline ISMO & C01DA08 & 0.35 & 2.90 & 0.34 & 2.79 & 0.43 & 3.58 & 0.33 & 2.70 \\
\hline Lisinopril & C09AA03 & 0.05 & 0.75 & 0.08 & 1.36 & 0.09 & 1.50 & 0.04 & 0.62 \\
\hline Losartan & C09CA01 & 0.33 & 5.52 & 0.30 & 4.89 & 0.35 & 5.79 & 0.32 & 5.19 \\
\hline Metoprolol & C07AB02 & 0.63 & 3.45 & 0.62 & 3.36 & 0.68 & 3.75 & 0.59 & 3.23 \\
\hline Mexilitine & C01BB02 & 0.16 & 0.49 & 0.14 & 0.42 & 0.16 & 0.51 & 0.14 & 0.43 \\
\hline Nifedipine & C08CA05 & 0.16 & 10.36 & 0.17 & 11.45 & 0.16 & 10.64 & 0.15 & 9.96 \\
\hline Pentoxiphylline & C04AD03 & 0.16 & 0.31 & 0.18 & 0.35 & 0.16 & 0.33 & 0.15 & 0.29 \\
\hline Prazosin & C02CA01 & 0.22 & 1.47 & 0.23 & 1.48 & 0.25 & 1.64 & 0.21 & 1.41 \\
\hline Propranolol & C06AA05 & 0.93 & 3.58 & 0.92 & 3.51 & 0.92 & 3.58 & 0.93 & 3.55 \\
\hline Ramipril & C09AA05 & 1.61 & 26.59 & 1.60 & 26.20 & 1.68 & 27.95 & 1.60 & 26.29 \\
\hline Ticlopidine & B01AC05 & 0.33 & 5.52 & 0.29 & 4.67 & 0.33 & 5.52 & 0.29 & 4.84 \\
\hline Verapamil & C08DA01 & 0.06 & 0.32 & 0.17 & 0.90 & 0.07 & 0.39 & 0.06 & 0.32 \\
\hline \multicolumn{10}{|c|}{ Category: Musculoskeletal } \\
\hline ASA & N02BA01 & 1.93 & 22.39 & 1.93 & 22.14 & 1.95 & 22.67 & 2.00 & 22.96 \\
\hline Celecoxib & M01AH01 & 0.25 & 1.66 & 0.29 & 1.93 & 0.27 & 1.77 & 0.28 & 1.83 \\
\hline Diclofenac sodium & M01AB05 & 2.42 & 40.02 & 0.29 & 37.50 & 2.41 & 40.02 & 2.50 & 41.10 \\
\hline HCQS & P01BA02 & 0.09 & 1.34 & 0.11 & 1.69 & 0.09 & 1.34 & 0.11 & 1.61 \\
\hline Ibuprofen & M01AE01 & 2.12 & 35.05 & 2.16 & 35.32 & 2.35 & 39.13 & 2.27 & 37.37 \\
\hline Indomethacin & M01AB01 & 0.16 & 1.17 & 0.16 & 1.19 & 0.15 & 1.14 & 0.19 & 1.40 \\
\hline Ketorolac & M01AB15 & 0.06 & 0.21 & 0.07 & 0.25 & 0.06 & 0.21 & 0.06 & 0.23 \\
\hline Mefenamic acid & M01AG01 & 0.14 & 0.84 & 0.13 & 0.76 & 0.13 & 0.74 & 0.16 & 0.90 \\
\hline Meloxicam & M01AC06 & 0.68 & 3.73 & 0.66 & 3.59 & 0.65 & 3.59 & 0.72 & 3.97 \\
\hline Naproxen & M01AE02 & 0.51 & 3.95 & 0.52 & 4.01 & 0.53 & 4.10 & 0.55 & 4.20 \\
\hline Paracetamol & N02BE01 & 2.40 & 19.91 & 2.43 & 19.92 & 2.41 & 20.01 & 2.48 & 20.34 \\
\hline Piroxicam & M01AC01 & 0.51 & 3.95 & 0.46 & 3.55 & 0.54 & 4.20 & 0.55 & 4.20 \\
\hline
\end{tabular}


Table 9: Contd...

\begin{tabular}{|c|c|c|c|c|c|c|c|c|c|}
\hline \multirow[t]{2}{*}{ Drug } & \multirow[t]{2}{*}{ ATC } & \multicolumn{2}{|c|}{ Jan-Mar } & \multicolumn{2}{|c|}{ Apr-Jun } & \multicolumn{2}{|c|}{ Jul-Sep } & \multicolumn{2}{|c|}{ Oct-Dec } \\
\hline & & $\%$ & DDD & $\%$ & DDD & $\%$ & DDD & $\%$ & DDD \\
\hline Rofecoxib & M01AH02 & 1.52 & 11.74 & 0.74 & 5.62 & 1.50 & 11.68 & 1.52 & 11.66 \\
\hline \multicolumn{10}{|l|}{ Category: AMA } \\
\hline Acyclovir & J05AB01 & 0.06 & 0.57 & 0.06 & 0.63 & 0.07 & 0.74 & 0.06 & 0.58 \\
\hline Albendazole & P02CA03 & 0.04 & 0.44 & 0.38 & 0.41 & 0.41 & 0.45 & 0.40 & 0.44 \\
\hline Amantadine & N04BB01 & 0.17 & 2.80 & 0.16 & 2.64 & 0.16 & 2.73 & 0.17 & 2.77 \\
\hline Amoxicillin & J01CA04 & 1.05 & 12.99 & 1.08 & 13.27 & 1.05 & 13.04 & 1.05 & 12.97 \\
\hline Azithromycin & L01FA10 & 0.07 & 0.55 & 0.12 & 0.98 & 0.08 & 0.65 & 0.06 & 0.52 \\
\hline Cephalexin & J01DA01 & 0.06 & 0.36 & 0.09 & 0.56 & 0.06 & 0.36 & 0.07 & 0.42 \\
\hline Chloramphenicol & J01BA01 & 0.04 & 0.10 & 0.04 & 0.09 & 0.02 & 0.05 & 0.04 & 0.11 \\
\hline Chloroquine & P01BA01 & 0.28 & 4.70 & 0.32 & 5.27 & 0.31 & 5.11 & 0.28 & 4.57 \\
\hline Ciprofloxacin & J01MA02 & 0.87 & 10.12 & 0.92 & 10.54 & 0.89 & 10.40 & 0.88 & 10.17 \\
\hline Clofazimine & J04BA01 & 0.26 & 12.68 & 0.28 & 13.55 & 0.28 & 13.91 & 0.26 & 12.87 \\
\hline Cloxacillin & J01CF02 & 0.31 & 1.30 & 0.34 & 1.39 & 0.32 & 1.33 & 0.32 & 1.30 \\
\hline Dapsone & J04BA02 & 0.27 & 8.86 & 0.26 & 8.43 & 0.28 & 9.27 & 0.27 & 9.00 \\
\hline DEC & P02CB02 & 0.16 & 1.36 & 0.16 & 1.38 & 0.16 & 1.36 & 0.16 & 1.38 \\
\hline Doxycyline & J01AA02 & 0.77 & 17.00 & 0.77 & 16.87 & 0.77 & 17.00 & 0.86 & 18.91 \\
\hline Erythromycin & J01FA01 & 0.40 & 1.56 & 0.44 & 1.67 & 0.40 & 1.54 & 0.46 & 1.78 \\
\hline Ethambutol & J04AK02 & 0.27 & 4.43 & 0.30 & 4.97 & 0.27 & 4.50 & 0.32 & 5.19 \\
\hline Ethionamide & J04AD03 & 0.06 & 1.91 & 0.09 & 2.86 & 0.05 & 1.50 & 0.08 & 2.77 \\
\hline Fluconazole & J02AC01 & 0.16 & 0.22 & 0.16 & 0.21 & 0.06 & 0.08 & 0.16 & 0.22 \\
\hline Furazolidone & G01AX06 & 0.09 & 1.43 & 0.16 & 2.64 & 0.09 & 1.43 & 0.11 & 1.80 \\
\hline Griseofulvin & D01BA01 & 0.47 & 5.44 & 0.53 & 6.11 & 0.46 & 5.34 & 0.51 & 5.91 \\
\hline Isoniazid & J04AC01 & 0.13 & 4.23 & 0.21 & 6.78 & 0.12 & 3.95 & 0.15 & 4.84 \\
\hline Ketoconazole & J02AB02 & 0.08 & 1.30 & 0.10 & 1.66 & 0.09 & 1.43 & 0.08 & 1.38 \\
\hline Levofloxacin & J01MA12 & 0.20 & 4.68 & 0.20 & 4.64 & 0.21 & 4.77 & 0.22 & 5.04 \\
\hline Metronidazole & P01AB01 & 0.63 & 2.94 & 0.62 & 2.83 & 0.61 & 2.83 & 0.69 & 3.20 \\
\hline Minocycline & $\mathrm{A} 01 \mathrm{AB} 23$ & 0.26 & 4.07 & 0.26 & 3.94 & 0.29 & 4.45 & 0.29 & 4.46 \\
\hline Nalidixic acid & J01MB02 & 0.40 & 2.31 & 0.39 & 2.21 & 0.43 & 2.51 & 0.46 & 2.64 \\
\hline Nitrofurantoin & J01XE01 & 0.28 & 3.25 & 0.29 & 3.37 & 0.32 & 3.67 & 0.29 & 3.34 \\
\hline Norfloxacin & J01MA06 & 0.87 & 13.43 & 0.87 & 13.28 & 0.92 & 14.32 & 0.95 & 14.53 \\
\hline Ofloxacin & J01MA01 & 0.19 & 1.02 & 0.18 & 0.98 & 0.21 & 1.16 & 0.21 & 1.13 \\
\hline Pefloxacin & J01MA03 & 0.18 & 1.40 & 0.6 & 1.23 & 0.17 & 1.34 & 0.20 & 1.55 \\
\hline Primaquine & P01BA03 & 0.16 & 0.21 & 0.16 & 0.20 & 0.16 & 0.20 & 0.16 & 0.21 \\
\hline Pyrazinamide & J04AK01 & 0.15 & 2.52 & 0.15 & 2.48 & 0.16 & 2.73 & 0.17 & 2.77 \\
\hline Quinine & P01BC01 & 0.12 & 1.07 & 0.17 & 1.60 & 0.16 & 1.45 & 0.14 & 1.32 \\
\hline Rifampicin & J04AB02 & 0.35 & 8.69 & 0.34 & 8.47 & 0.37 & 9.20 & 0.37 & 9.13 \\
\hline Roxitromycin & J01FA06 & 0.27 & 2.07 & 0.25 & 1.90 & 0.28 & 2.16 & 0.27 & 2.10 \\
\hline Secnidazole & P02AB07 & 0.12 & 0.38 & 0.13 & 0.42 & 0.12 & 0.40 & 0.13 & 0.42 \\
\hline Sulfamethoxazole & J01EC01 & 0.26 & 1.22 & 0.22 & 1.01 & 0.30 & 1.37 & 0.29 & 1.34 \\
\hline Tinidazole & P01AB02 & 0.55 & 2.13 & 0.52 & 2.00 & 0.55 & 2.15 & 0.59 & 2.26 \\
\hline \multicolumn{10}{|l|}{ Category: CNS } \\
\hline Alprazolam & N05BA12 & 0.19 & 0.72 & 0.17 & 0.63 & 0.21 & 0.80 & 0.25 & 0.95 \\
\hline Amitryptilline & N06AA09 & 0.06 & 0.41 & 0.05 & 0.33 & 0.06 & 0.41 & 0.10 & 0.66 \\
\hline Baclofen & M03BX01 & 0.32 & 2.13 & 0.30 & 1.96 & 0.31 & 2.05 & 0.40 & 2.63 \\
\hline Betahistine & N07CA01 & 0.30 & 5.05 & 0.30 & 4.89 & 0.35 & 5.79 & 0.39 & 6.37 \\
\hline Bromocriptine & N04BC01 & 0.16 & 0.91 & 0.17 & 1.00 & 0.20 & 1.17 & 0.29 & 1.65 \\
\hline
\end{tabular}


Table 9: Contd...

\begin{tabular}{|c|c|c|c|c|c|c|c|c|c|}
\hline \multirow[t]{2}{*}{ Drug } & \multirow[t]{2}{*}{ ATC } & \multicolumn{2}{|c|}{ Jan-Mar } & \multicolumn{2}{|c|}{ Apr-Jun } & \multicolumn{2}{|c|}{ Jul-Sep } & \multicolumn{2}{|c|}{ Oct-Dec } \\
\hline & & $\%$ & DDD & $\%$ & DDD & $\%$ & DDD & $\%$ & DDD \\
\hline Buspirone & N05BE01 & 0.03 & 0.18 & 0.04 & 0.20 & 0.06 & 0.34 & 0.06 & 0.35 \\
\hline Carbamazepine & N03AF01 & 0.73 & 7.28 & 0.71 & 6.96 & 0.82 & 8.22 & 0.18 & 1.74 \\
\hline Chlordiazepoxide & N05BA02 & 0.19 & 2.05 & 0.17 & 1.81 & 0.23 & 2.50 & 0.27 & 3.00 \\
\hline Buspirone & N05BE01 & 0.03 & 0.18 & 0.04 & 0.20 & 0.06 & 0.34 & 0.06 & 0.35 \\
\hline Carbamazepine & N03AF01 & 0.73 & 7.28 & 0.71 & 6.96 & 0.82 & 8.22 & 0.18 & 1.74 \\
\hline Chlordiazepoxide & N05BA02 & 0.19 & 2.05 & 0.17 & 1.81 & 0.23 & 2.50 & 0.27 & 3.00 \\
\hline Cinnarizine & N07CA02 & 0.06 & 0.49 & 0.08 & 0.70 & 0.11 & 0.99 & 0.08 & 0.68 \\
\hline Citalopram & N06AB04 & 0.08 & 1.94 & 0.09 & 2.15 & 0.10 & 2.45 & 0.11 & 2.70 \\
\hline Clobazam & N05BA09 & 0.63 & 5.25 & 0.63 & 5.20 & 0.71 & 5.86 & 0.80 & 6.54 \\
\hline Clomipramine & N06AA04 & 0.27 & 2.66 & 0.27 & 2.62 & 0.32 & 3.19 & 0.40 & 3.90 \\
\hline Clonazepam & N03AE01 & 0.10 & 0.41 & 0.09 & 0.38 & 0.14 & 0.58 & 0.17 & 0.69 \\
\hline Clozapine & N05AH02 & 0.07 & 0.31 & 0.07 & 0.28 & 0.10 & 0.43 & 0.13 & 0.55 \\
\hline Diazepam & N05BA01 & 0.28 & 4.64 & 0.30 & 4.97 & 0.28 & 4.70 & 0.36 & 5.88 \\
\hline Doxipine & N06AA12 & 0.77 & 0.22 & 0.05 & 0.17 & 0.10 & 0.33 & 0.13 & 0.43 \\
\hline Ergotamine & N02CA02 & 0.07 & 0.51 & 0.07 & 0.53 & 0.10 & 0.76 & 0.13 & 1.00 \\
\hline Flunarizine & N07CA03 & 0.47 & 7.77 & 0.44 & 7.23 & 0.51 & 8.45 & 0.61 & 10.03 \\
\hline Fluoxetine & N06AB03 & 0.33 & 16.16 & 0.34 & 16.49 & 0.34 & 17.18 & 0.48 & 23.46 \\
\hline Fluvoxamine & N06AB08 & 0.21 & 3.55 & 0.20 & 3.31 & 0.22 & 3.68 & 0.27 & 4.50 \\
\hline Haloperidol & N05AD01 & 0.14 & 0.89 & 0.20 & 1.24 & 0.17 & 1.07 & 0.23 & 1.43 \\
\hline Chlorpromazine & N05AA01 & 0.22 & 2.41 & 0.23 & 2.46 & 0.24 & 2.64 & 0.33 & 3.60 \\
\hline Levodopa & N04BA02 & 0.36 & 5.93 & 0.36 & 5.87 & 0.38 & 6.34 & 0.46 & 7.61 \\
\hline Lithium carbonate & N05AN01 & 0.26 & 8.73 & 0.30 & 9.94 & 0.27 & 9.00 & 0.35 & 11.62 \\
\hline Lorazepam & N05BA06 & 0.27 & 3.55 & 0.30 & 1.99 & 0.26 & 1.75 & 0.036 & 9.52 \\
\hline Nimodipine & C08CA06 & 0.22 & 0.74 & 0.27 & 0.89 & 0.24 & 0.79 & 0.30 & 0.50 \\
\hline Nitrazepam & N05CD02 & 0.21 & 10.64 & 0.25 & 12.42 & 0.23 & 11.45 & 0.29 & 14.12 \\
\hline Nortriptyline & N06AA10 & 0.27 & 2.95 & 0.27 & 2.91 & 0.22 & 2.24 & 0.35 & 3.78 \\
\hline Olanzapine & N05AH03 & 0.09 & 0.72 & 0.11 & 0.87 & 0.10 & 0.85 & 0.15 & 1.21 \\
\hline Phenobarbitone & N03AA02 & 0.27 & 5.32 & 0.28 & 5.42 & 0.29 & 5.73 & 0.34 & 6.64 \\
\hline Phenytoin sodium & N03AB02 & 0.20 & 3.27 & 0.25 & 4.07 & 0.21 & 3.48 & 0.25 & 4.08 \\
\hline Risperidone & N05AX08 & 0.27 & 2.66 & 0.27 & 2.62 & 0.25 & 2.50 & 0.34 & 3.32 \\
\hline Selegiline & N04BD01 & 0.20 & 9.82 & 0.23 & 11.07 & 0.21 & 10.23 & 0.34 & 16.81 \\
\hline Sertraline & N05AE03 & 0.24 & 12.57 & 0.27 & 13.88 & 0.19 & 10.01 & 0.27 & 13.84 \\
\hline Sodium valproate & N03AG01 & 0.52 & 3.21 & 2.50 & 3.04 & 0.46 & 2.85 & 0.67 & 4.13 \\
\hline Pyridostigmine & N07AA02 & 0.26 & 2.82 & 0.31 & 3.41 & 0.30 & 3.36 & 0.38 & 4.20 \\
\hline Zolpidem & N05CF02 & 0.23 & 7.64 & 0.25 & 8.28 & 0.23 & 7.50 & 0.34 & 11.07 \\
\hline \multicolumn{10}{|l|}{ Category: GIT } \\
\hline Cisapride & A03FA02 & 0.06 & 0.76 & 0.21 & 2.71 & 0.06 & 0.76 & 0.08 & 1.11 \\
\hline Colloidal bismuth subcitrate & A02BX05 & 0.12 & 1.48 & 0.34 & 4.24 & 0.14 & 1.74 & 0.18 & 2.18 \\
\hline Digene & A02AD01 & 1.23 & 25.08 & 1.54 & 31.41 & 1.23 & 29.37 & 1.40 & 27.22 \\
\hline Diloxanide furoate & P01AC01 & 0.32 & 5.32 & 0.62 & 10.09 & 0.33 & 5.45 & 0.38 & 6.23 \\
\hline Domperidone & A03FA03 & 0.11 & 0.86 & 0.64 & 2.64 & 0.16 & 1.21 & 0.18 & 1.36 \\
\hline Esmoprazole & $\mathrm{A} 02 \mathrm{BC} 05$ & 0.63 & 9.80 & 0.91 & 13.85 & 0.73 & 11.33 & 0.81 & 12.40 \\
\hline Famotidine & A02BA03 & 0.61 & 10.09 & 0.80 & 1318 & 0.72 & 11.93 & 0.74 & 12.11 \\
\hline Flavonoids & C05CA01 & 0.14 & - & 0.34 & - & 0.20 & - & 0.20 & - \\
\hline Lansoprazole & A02BC03 & 0.15 & 8.45 & 0.76 & 12.42 & 0.57 & 9.41 & 0.55 & 9.00 \\
\hline Loperamide & A07DA03 & 0.02 & 0.15 & 0.05 & 0.28 & 0.02 & 0.15 & 0.03 & 0.21 \\
\hline
\end{tabular}


Table 9: Contd...

\begin{tabular}{|c|c|c|c|c|c|c|c|c|c|}
\hline \multirow[t]{2}{*}{ Drug } & \multirow[t]{2}{*}{ ATC } & \multicolumn{2}{|c|}{ Jan-Mar } & \multicolumn{2}{|c|}{ Apr-Jun } & \multicolumn{2}{|c|}{ Jul-Sep } & \multicolumn{2}{|c|}{ Oct-Dec } \\
\hline & & $\%$ & DDD & $\%$ & DDD & $\%$ & DDD & $\%$ & DDD \\
\hline Metoclopramide & AO3FA01 & 0.32 & 5.32 & 0.52 & 8.43 & 0.41 & 6.82 & 0.37 & 6.02 \\
\hline Mosapride & - & 0.55 & 6.09 & 0.72 & 7.83 & 0.68 & 7.50 & 0.58 & 6.37 \\
\hline Omeprazole & $\mathrm{A} 02 \mathrm{BC} 01$ & 1.59 & 18.47 & 1.78 & 20.40 & 1.68 & 19.57 & 1.64 & 18.84 \\
\hline Ondansetron & A04AA01 & 0.07 & 0.92 & 0.12 & 1.47 & 0.09 & 1.12 & 0.11 & 1.30 \\
\hline Prochlorperazine & NO5AB04 & 0.27 & 1.33 & 0.35 & 1.72 & 0.32 & 1.62 & 0.32 & 1.56 \\
\hline Ranitidine & A02BA02 & 1.15 & 57.28 & 1.44 & 70.93 & 1.30 & 65.04 & 1.26 & 62.28 \\
\hline Salazopyrine & A07EC01 & 0.19 & 2.30 & 0.30 & 3.67 & 0.24 & 2.97 & 0.23 & 2.85 \\
\hline \multicolumn{10}{|c|}{$\begin{array}{l}\text { Category: Respiratory system and } \\
\text { anti-allergics }\end{array}$} \\
\hline Bromhexine & R05CB02 & 0.63 & 6.53 & 0.76 & 7.73 & 0.63 & 6.49 & 0.65 & 6.63 \\
\hline Etophylline and theophylline & R03DA05 & 1.60 & 12.35 & 1.66 & 12.65 & 1.53 & 11.49 & 1.63 & 12.46 \\
\hline Salbutamol & $\mathrm{R} 03 \mathrm{CC} 02$ & 1.02 & 7.89 & 1.03 & 7.84 & 0.98 & 7.57 & 1.00 & 7.69 \\
\hline Astemizole & R06AX11 & 0.68 & 5.25 & 0.61 & 4.67 & 0.62 & 4.84 & 0.67 & 5.17 \\
\hline Cetrizine & R06AE0 & 0.93 & 7.16 & 0.98 & 7.52 & 0.83 & 6.43 & 0.90 & 6.91 \\
\hline Ketotifen & R06AX17 & 1.09 & 8.43 & 1.11 & 8.47 & 1.01 & 7.86 & 1.07 & 8.20 \\
\hline Terfenadine & R06AX12 & 1.13 & 8.75 & 1.22 & 9.31 & 1.15 & 8.91 & 1.13 & 8.65 \\
\hline Pheniramine maleate & R06AB06 & 1.37 & 10.60 & 1.38 & 10.54 & 1.29 & 9.99 & 1.35 & 10.37 \\
\hline Cyproheptadine & R06AX02 & 1.15 & 8.88 & 1.18 & 9.03 & 1.03 & 7.99 & 1.14 & 8.72 \\
\hline Promethazine & R06AD02 & 1.22 & 11.30 & 1.40 & 12.86 & 1.26 & 11.76 & 1.16 & 10.66 \\
\hline \multicolumn{10}{|c|}{ Category: Hormones and related drugs } \\
\hline Acarbose & A10BF01 & 0.28 & 2.28 & 0.23 & 1.88 & 0.33 & 2.73 & 0.27 & 2.21 \\
\hline Clomiphene citrate & G03GB02 & 0.19 & 2.99 & 0.16 & 2.46 & 0.27 & 4.14 & 0.19 & 2.19 \\
\hline Conjugated estrogen & G03CA57 & 0.63 & 14.70 & 0.57 & 13.18 & 0.73 & 16.89 & 0.65 & 15.02 \\
\hline Danazole & G03XA01 & 0.63 & 5.25 & 0.62 & 5.08 & 0.72 & 5.97 & 0.62 & 5.12 \\
\hline Dexamethasone & H02AB02 & 0.69 & 5.35 & 0.63 & 4.78 & 0.76 & 5.89 & 0.62 & 4.78 \\
\hline Eltroxine & H03AA01 & 0.49 & 10.91 & 0.41 & 9.04 & 0.58 & 12.91 & 0.43 & 9.50 \\
\hline Glibenclamide & A10BB01 & 1.29 & 14.99 & 1.27 & 14.60 & 1.41 & 16.46 & 1.26 & 14.53 \\
\hline Gliclazide & A10BB09 & 0.63 & 7.30 & 0.57 & 6.59 & 0.72 & 8.35 & 0.61 & 7.02 \\
\hline Glimepride & $\mathrm{A} 10 \mathrm{BB} 12$ & 0.51 & 12.68 & 0.51 & 12.42 & 0.59 & 14.62 & 0.47 & 11.52 \\
\hline Glipizide & A10BB07 & 1.05 & 12.99 & 0.99 & 12.14 & 1.13 & 14.06 & 0.90 & 11.11 \\
\hline Metformin & A01BA02 & 1.06 & 26.29 & 0.92 & 22.70 & 1.17 & 29.25 & 0.95 & 23.35 \\
\hline Neomercazole & Н03ВВ01 & 0.56 & 9.20 & 0.57 & 9.34 & 0.65 & 10.84 & 0.53 & 8.65 \\
\hline Norethisterone & G03AC01 & 0.09 & 1.34 & 0.09 & 1.41 & 0.11 & 1.78 & 0.08 & 1.23 \\
\hline Pioglitazone & A10BG03 & 0.16 & 2.42 & 0.14 & 2.18 & 0.20 & 3.05 & 0.14 & 2.13 \\
\hline Prednisolone & H02АB06 & 0.36 & 3.95 & 0.32 & 3.51 & 0.41 & 4.55 & 0.31 & 3.41 \\
\hline Repaglinide & A10BX02 & 0.22 & 0.86 & 0.21 & 0.79 & 0.27 & 1.03 & 0.19 & 0.73 \\
\hline Rosiglitazone & A10BG02 & 0.58 & 6.45 & 0.57 & 6.22 & 0.61 & 6.73 & 0.53 & 5.77 \\
\hline \multicolumn{10}{|l|}{ Category: Vitamins and nutrition } \\
\hline Calcium carbonate & A12AA03 & 2.69 & 5.95 & 2.33 & 5.09 & 2.69 & 5.97 & 2.67 & 5.86 \\
\hline Ferrous sulphate & B03AA07 & 2.87 & 47.59 & 2.31 & 37.80 & 2.05 & 34.02 & 2.30 & 37.78 \\
\hline Alfacalcidiol & M05A12A & 0.58 & 2.26 & 0.57 & 2.18 & 0.39 & 1.51 & 0.41 & 1.58 \\
\hline B complex & A11EB03 & 3.90 & - & 3.62 & - & 3.26 & - & 3.24 & - \\
\hline Folic acid & B03BB01 & 2.26 & 34.87 & 1.79 & 27.41 & 1.55 & 24.12 & 1.40 & 21.51 \\
\hline Vitamin C & A11GA01 & 1.95 & 10.75 & 1.72 & 9.41 & 1.37 & 7.57 & 1.21 & 6.64 \\
\hline Vitamin E & A11HA03 & 1.91 & 44.39 & 1.83 & 41.96 & 1.56 & 36.27 & 1.25 & 28.87 \\
\hline
\end{tabular}

Contd... 
Table 9: Contd...

\begin{tabular}{|c|c|c|c|c|c|c|c|c|c|}
\hline \multirow[t]{2}{*}{ Drug } & \multirow[t]{2}{*}{ ATC } & \multicolumn{2}{|c|}{ Jan-Mar } & \multicolumn{2}{|c|}{ Apr-Jun } & \multicolumn{2}{|c|}{ Jul-Sep } & \multicolumn{2}{|c|}{ Oct-Dec } \\
\hline & & $\%$ & DDD & $\%$ & DDD & $\%$ & DDD & $\%$ & DDD \\
\hline \multicolumn{10}{|c|}{ Category: Metabolism } \\
\hline Alendronate & M05BA04 & 1.23 & 13.64 & 1.14 & 12.40 & 1.23 & 13.64 & 1.13 & 12.41 \\
\hline Atorvastatin & C10AA05 & 1.58 & 52.37 & 1.40 & 45.78 & 1.58 & 52.50 & 1.51 & 49.54 \\
\hline Gemfibrozil & C10AB04 & 1.63 & 18.90 & 1.43 & 16.45 & 1.62 & 18.85 & 1.53 & 17.63 \\
\hline Lovastatin & C10AA02 & 1.59 & 17.59 & 1.54 & 16.82 & 1.58 & 17.50 & 1.47 & 16.05 \\
\hline Simvastatin & C10AA01 & 2.46 & 54.37 & 2.15 & 46.99 & 2.53 & 55.99 & 2.32 & 50.74 \\
\hline UDCA & A5AA02 & 1.43 & 33.22 & 1.36 & 31.10 & 1.63 & 37.89 & 1.37 & 31.48 \\
\hline
\end{tabular}

CVS: Cardiovascular, GIT: Gastrointestinal, CNS: Central nervous system, UDCA: Ursodeoxycholic acid

below two but higher figure is always fraught with increased risk of drug interaction and errors of prescribing.

The increase in a number of patients with advancing age especially above 50-year suggests a need of time to address the requirement of the geriatric population separately. A voluminous increase of prescription for drug groups like - CVS, nutrition and vitamins, metabolic drugs, hormones and related drugs, NSAIDs, psychotropics, and AMAs is pointer to prevailing morbidity pattern of society. There is a sharp decrease in no. of patients $>70$ year age group probably due to age-related in-capabilities to collect medicines at the hospital dispensary.

Between the genders, common group of drugs highly prescribed to both are from nutrition and vitamins. However, the second highest drug prescribed for males has been CVS drugs and likewise for females been NSAIDs. Furthermore, these findings suggest of high prevalence of CVS related morbidity such as MI, CAD, and hypertension prevalent in the male subpopulation and females of pain related ailments.

Pharmacoeconimically, the budget spent in all four quarters of the period of study, suggests more or less a uniform pattern without significant variation. Among the various drug groups, the order of the expense has been on metabolic drugs, AMAs, and hormones and related drugs. Majority of such drug groups are recently been available on the shelf and are of specific needs of the elderly population. This indicates a rising trend of metabolic disorders as well as endocrinal disorders, all pointers toward diseases of sedentary lifestyles with reduced fitness levels. AMAs per say are not the only one with a high cost per tablet but also been the most misused and over prescribed group of drugs.

The basic definition of the DDD is: The assumed average maintenance dose per day for a drug used for its main indication in adults. ${ }^{29}$ The DDD methodology does not indicate the exact number of patients who have been treated with a drug. This concept assumes that every person prescribed a particular drug is taking the specified dose of the said drug every day, ignoring alterations of dose of the drug by disease or if any patient-related factors be there or any other variable. With this concept of drug utilization, a drawback may be that drugs prescribed for a brief period can have their prescribing prevalence underestimated too. Variation in the pattern of dosage, duration of the study, the scatter of population over different ages, gender and races, all can contribute to differences in audit reports using DDD methodology to determine the prevalence of prescribing.

An important basis for the WHO recommendation to use the ATC/DDD methodology in drug utilization studies is to improve comparison of drug utilization studies in making international comparisons and to study long-term trends in drug use. Due to continuous change and development of the drug assortment and drug uses, a regular revision of the ATC system is also necessary. Some ATC and DDD alterations are made annually, and an updated version of the list that includes all officially assigned ATC codes and DDDs is issued every year. ${ }^{29}$ In order to compare data from different countries and different periods, it is essential to know which ATC codes and DDDs are used. Consumption figures may look very different when different ATC/DDD versions are applied.

To have uniformity in drug utilization studies world over, DDD is of great value as compared to any traditional method of use determination like in percentage, or weight or cost wise. Study of drug utilization in India has been reported both in hospital ${ }^{19-21}$ and community levels..$^{22,23,27}$ Most of these studies have measured drug utilization by counting of prescriptions which actually indicates the prescribing frequency. The study of drug utilization is made more meaningful if the frequency of prescribing is studied with a total quantity of drugs per prescription. Indian studies have used DDD concept and focused on drug utilization in selected groups of patients for a brief period, ${ }^{24-26}$ whereas this study was undertaken the study on a vast scale for the first time the country.

Drugs were classified using ATC classification system which mandates the drugs to divided into different groups according to the organ or system on which they act and their chemical, pharmacological and therapeutic property. ${ }^{28}$ Drugs are classified in groups at five different levels. There are fourteen main groups (first level), with two therapeutic/ 
pharmacological subgroups (second and third levels), the fourth level is a therapeutic/pharmacological chemical subgroup, and the fifth level is the chemical substance.

The complete classification of metformin illustrates the structure of the code:

A: Alimentary tract and metabolism (first level, anatomical main group).

A10: Drugs used in diabetes (second level, pharmacological/ therapeutic main group).

A10B: Oral blood glucose lowering drugs (third level, chemical/pharmacological/therapeutic subgroup).

A1013 A: Biguanides (fourth level, chemical/ pharmacoiogical/therapeutic subgroup).

A10BA02: Metíormin (fifth level, subgroup for the chemical substance).

Thus, in the ATC system, all plain metformin preparations are given the code A10BA02. Prescribing prevalence studies are also useful to determine the prevailing morbidity patterns. To quote an example from our study, the DDD/1000/day of amlodipine (49.77), frusemide (40.09), and ramipril (26.59) in the first quarter, and likewise high value in all other quarters of year points toward a pandemic state of CVS disorders like hypertension and myocardial ischemia, etc. Similarly, the high prescription pattern for antipsychotics like fluoxetine (16.16), sertraline (12.57), nitrazepam (10.64) is an index of high psychiatric morbidity in the society. Also in a drug group of GIT, it is realized a high consumption pattern of ranitidine (57.28) and likewise in the other quarter of years too, is an indicator toward urbanization and its related disorders of anxiety and poor hygiene sanitation. A high usage of OHAs like metformin (26.29), glimepride (12.68), glipizide (12.99), and metabolic drugs like atorvastatin (52.37), simvastatin (54.37) are indicators toward growing prevalence of metabolic disorders in the society.

Studies elsewhere in India on prescription audit employing the DDD concept ${ }^{24-26}$ have focused on a select group of patients which could not be meaningfully compared to our data because the present study covers a general outpatient department (OPD) population. Present study is done on a sample population of an urban demographic profile dependent for their health care needs through OPDs on a government tertiary care hospital. This may be giving insight into the day to day functioning status of our health care delivery system and opportunity on self-assessment in further improving the delivery of health care by implementing measures which economizes on scarce health care budget and facility and optimize the needful help to patients by minimizing errors in prescription writing by physicians. This study may serve as one of the works upon which further studies in prescription audit can be taken up to improve the scope for improvement in prescribing practices among the physicians.

Funding: No funding sources

Conflict of interest: None declared

Ethical approval: The study was approved by the Institutional Ethical Committee

\section{REFERENCES}

1. Millard A. Perceptions of clinical audit: A preliminary evaluation. J Clin Eff. 1996;1(3):96-9.

2. Hopkins A. Clinical audit: time for a reappraisal? J R Coll Physicians Lond. 1996;30(5):415-25.

3. Robinson S. Evaluating the progress of clinical audit. Int J Theory Res Pract. 1996;2:373-92.

4. Duke MNG. Drug Utilization Studies-Method and Uses. European Series No. 45. Copenhagen, Denmark: WHO Regional Publications; 1993. p. 1-4.

5. Rønning M. Coding and classification in drug statistics - From national to global application. Nor J Epidemiol. 2001;11(1):37-40.

6. Ramsay LE. Bridging the gap between clinical pharmacology and rational drug prescribing. $\mathrm{Br} \mathrm{J}$ Clin Pharmacol. 1993;35(6):575-6.

7. Gupta N, Sharma D, Garg SK, Bhargava VK. Auditing of prescriptions to study utilization of antimicrobials in a tertiary hospital. Indian J Pharmacol. 1997;29(6):411-15.

8. Lord J, Johns LP. Development of an instrument to assess staff perceptions of the impact of trust-based clinical audit programmes. J Clin Eff. 1996;1(3):83-9.

9. Robinson S. Audit in the therapy professions: Some constraints on progress. Qual Health Care. 1996;5(4):206-14.

10. Curtis P. Medical audit in general practice. J R Coll Gen Pract. 1974;24(146):607-11.

11. Gaud RS, Jain DK, Kaskhedikar SG, Chaturvedi SC. Critical evaluation of present prescribing pattern. Indian J Hosp Pharm. 1989:26:70-72.

12. Benet LZ. Principles of prescription order writing and patients compliance instructions. In: Goodman AG, Rail TW, Nies AS, Taylor P, editors. Goodman and Gilman's The Pharmacological Basis of Therapeutics. $8^{\text {th }}$ edition. New York: Pegamon Press Inc.; 1991. p. 1640.

13. Shrishlya MV, Mahesh K, Nagarani MA, Sr Mary C, Andrade C, Venkataraman BV. Prescription audit study in an Indian hospital setting using the DDD (defined daily dose) concept. Indian J Pharmacol. 1994;26(1):23-8.

14. Patterson HR. The problems of audit and research. J R Coll Gen Pract. 1986;36(286): 196.

15. Ross-Degnan D, Laing R, Santoso B, Ofori-Adjei D, Lamoureux C, Hogerzeil H. Improving pharmaceutical use in primary care in developing countries: a critical review of experience and lack of experience. Paper Presented at the 1st International Conference on Improving the Use of Medicines, Chiang Mai, Thailand, 1-4 April, 1997.

16. Hogerzeil HV, Bimo, Ross-Degnan D, Laing RO, OforiAdjei D, Santoso B, et al. Field tests for rational drug use in twelve developing countries. Lancet. 1993;342(8884):1408-10.

17. Laing R, Hogerzeil H, Ross-Degnan D. Ten recommendations to improve use of medicines in developing countries. Health Policy Plan. 2001;16(1):13-20.

18. Reizenstein P. Quality and health care in Sweden. BMJ. 1991;303(6807):900-2. 
19. Kapoor B, Raina RK, Kapoor S. Drug prescribing pattern in teaching hospital unit. Indian $\mathrm{J}$ Pharmacol. 1985;17 Suppl 1:168.

20. Kumar H, Gupta U, Grag KC, Agarwal KK. A Study of trend of drug usage in hospital unit. Indian J Pharmacol. 1986:18:50.

21. Sood B, Verma RK, Gulati PV. Diagnosis and treatment in general hospital. Clinician 1984;48:263-70.

22. Hede SS, Diniz RS, Agshikar NV, Dhume VG. Pattern of prescribed and OTC drugs in North Goa. Indian J Pharmacol. 1987;19:145-8.

23. Krishnaswamy K, Kumar BD, Radhaiah G. A drug survey - Precepts and practices. Eur J Clin Pharmacol. 1985;29(3):363-70

24. Uppal R. Utilization of antimicrobials for caesarean section in a district hospital in India. Apua Newsl. 1990;8:2.

25. Uppal R. Antimicrobial drug usage in urology. Int J Clin Pharmacol Ther Toxiocol. 1991;29(a):336-8
26. Uppal R. Drug utilization of antimicrobials in caesarean section. J Obstet Gynecol India. 1991;41:346-9.

27. Jaju BP. Practicality of Alma Alta declaration: some observations in drug profile of prescriptions. Indian J Pharmacol. 1985; 17:229-32.

28. Guidelines for ATC Classification and DDD Assignment. Oslo, Norway: WHO Collaborating Centre for Drug Statistics Methodology; 2012.

29. ATC Index with DDDs. Oslo, Norway: WHO Collaborating Centre for Drug Statistics Methodology; 2013.

doi: 10.5455/2319-2003.ijbcp20141033

Cite this article as: Gupta AK, Mishra S. Prescription audit study in a tertiary care hospital using the anatomical therapeutic chemical and defined daily dose classification concept. Int J Basic Clin Pharmacol 2014;3:889-901. 